\title{
KN15 | Changing Times In StructuralVirology
}

Stuart, Dave (University of Oxford, Old Road Campus, GBR)

Macromolecular crystallography has provided results that underpin much biological discovery and there is still considerable scope for further development, however the recent revolution in electron imaging now means that it can also routinely provide detailed atomic level descriptions. Macromolecular crystallography has provided the pre-eminent example of fast, accurate, automated data collection at synchrotrons driving scientific discovery and democratising science, and that example is now inspiring the massive rate of change in electron imaging. I will try to present thoughts on where crystallography is going, but also to indicate how electron diffraction, electron microscopy and electron tomography are starting to open up otherwise inaccessible areas of structural biology. 